\title{
A Randomized, Controlled Trial of Topical Application of Virgin Coconut Oil in the Prevention of Nosocomial Infections in Neonates Born $\leq 34$ Weeks Gestational Age
}

\author{
Resti Ma. M. Bautista, ${ }^{1,2}$ Jacinto Blas V. Mantaring, IIII,3 and Anna Lisa Ong-Lim ${ }^{4}$ \\ ${ }^{1}$ Section of Newborn Medicine, Department of Pediatrics, College of Medicine and Philippine General Hospital, University of the Philippines Manila \\ Institute of Child Health and Human Development, National Institutes of Health, University of the Philippines Manila \\ ${ }^{3}$ Department of Clinical Epidemiology, College of Medicine, University of the Philippines Manila \\ ${ }^{4}$ Section of Infectious and Tropical Diseases, Department of Pediatrics, College of Medicine and Philippine General Hospital, University of the Philippines Manila
}

\begin{abstract}
Objective. To determine the efficacy of topically applied virgin coconut oil (TVCO) in the prevention of nosocomial infection in neonates born $\leq 34$ weeks gestational age.

Method. A prospective, randomized, non-blinded, controlled clinical trial was conducted at the Neonatal Intensive Care Unit (NICU) of the Philippine General Hospital. Preterm infants received TVCO $4 \mathrm{~g} / \mathrm{kg}$ twice daily for 14 days or routine skin care, and followed up until 28 days or discharge. Outcome measures included incidence of nosocomial infections, need for doublevolume exchange transfusion (DVET) for sepsis, all-cause mortality rates and length of hospital stay.
\end{abstract}

Results. After 52 infants were randomized, 24 to TVCO and 28 to routine care, the study was prematurely terminated due to a possibility of harm with an increased incidence of nosocomial pneumonia in the TVCO group. The groups were comparable in terms of baseline characteristics. There were no cases of meningitis, necrotizing enterocolitis and urinary tract infection in either group. The incidence of nosocomial sepsis in the TVCO group was $38 \%$ versus $43 \%$ in the routine care group ( $R R=0.875$ $[0.447,1.711] ; P=0.695)$. The incidence of nosocomial pneumonia was $17 \%$ in the TVCO group versus $7 \%$ in the control $(\mathrm{RR}=2.33$ $[0.468,11.644] ; P=0.284)$. Mortality rate in the TVCO group was $12 \%$ versus $14 \%$ in the control (RR=0.875 [0.217, 3.528]; $P=0.851)$. A trend toward decreased need for DVET for sepsis was found in infants treated with TVCO (RR=0.166 [0.009, 3.056]; $P=0.098)$. Length of hospital stay was not clinically different (Mean difference $=0.091$ days $[-2.338,2.520]$ ).

Conclusion. There was insufficient evidence to conclude there was a difference in the incidence of nosocomial infections, mortality rates and length of hospital stay between the two treatment groups. There was a trend for decreased need for DVET for sepsis in the TVCO group. However, there was possibility of harm with increased incidence of nosocomial pneumonia in the TVCO group. This clinical trial was stopped before the computed sample size was reached since the authors

Corresponding author: Resti Ma. M. Bautista, MD

Section of Newborn Medicine

Department of Pediatrics

Philippine General Hospital

University of the Philippines Manila

Taft Avenue, Ermita, Manila 1000 Philippines

Telephone: +6325240892

Email: restimd@gmail.com deemed it impractical and unethical to recruit more infants into the study given that the clinical benefits of TVCO in premature infants may not outweigh possible adverse outcomes. Future studies on the use of VCO would be better directed at evaluating effectiveness and safety of VCO given orally rather than via topical administration.

Key Words: virgin coconut oil, preterm, neonatal infection, neonatal sepsis

\section{Introduction}

Therapeutic options for managing infections in neonates in developing countries are often limited and thus, innovative approaches to preventing infections are needed. Topical therapy with skin barrier-enhancing products may be an effective strategy for improving neonatal outcomes, particularly among preterm, low birth weight infants whose skin barrier is temporarily but critically compromised as a result of immaturity. ${ }^{1}$ Topical therapy to enhance skin barrier function may be a simple, low-cost, effective strategy to improve outcome of preterm infants with a developmentally compromised epidermal barrier, as lipid constituents of topical products may act as a mechanical barrier and augment synthesis of barrier lipids. Natural oils are applied topically as part of a traditional oil massage to neonates in many developing countries $^{2}$ including the Philippines.

However, the safety of the use of topical ointments in premature infants has been questioned. Conner et al. in 2000 published a systematic review to assess the effect of prophylactic application of topical ointment on nosocomial sepsis rates and other complications of prematurity in preterm infants. Four randomized controlled trials were identified and reported improved skin condition in infants treated with prophylactic topical ointment. All four studies reported on the incidence of any nosocomial infection, fungal infection and coagulase negative staphylococcal infection. Infants treated with prophylactic topical ointment were at increased risk of coagulase negative staphylococcal infection (Relative risk 1.31, 95\% CI 1.02, 1.70; risk difference $0.04,95 \%$ CI $0.00,0.08$ ); and any nosocomial infection (Relative risk 1.20, 95\% CI 1.00, 1.43; risk difference 0.05, 95\% CI $0.00,0.09)$. A trend toward increased risk of any bacterial 
infection was found in infants treated with prophylactic topical ointment (Relative risk 1.19, 95\% CI 0.97, 1.46; risk difference $0.04,95 \%$ CI $-0.01,0.08)$. There was no significant difference found in the risk of bacterial infection with a known pathogen, fungal infection, or other complications related to prematurity. Their review concluded that prophylactic application of topical ointment increases the risk of coagulase negative staphylococcal infection and any nosocomial infection. A trend toward increased risk of any bacterial infection was noted in infants prophylactically treated. The review recommended that topical ointment should not be used routinely in preterm infants. ${ }^{3}$

A number of studies have explored alternatives for topical emollients for use in premature infants. Darmstadt et al. in 2002 aimed to identify inexpensive, safe, vegetable oils available in developing countries that improved epidermal barrier function. The impact of oils on mouse epidermal barrier function (rate of transepidermal water loss over time following acute barrier disruption by tape-stripping) and ultrastructure was determined. A single application of sunflower seed oil significantly accelerated skin barrier recovery within 1 hour; the effect was sustained 5 hours after application. Their report suggested that topical application of linoleate-enriched oil such as sunflower seed oil might enhance skin barrier function and improve outcome in neonates with compromised barrier function. ${ }^{2}$

In 2004, a randomized controlled investigation also by Darmstadt and colleagues, done in a NICU at Cairo University, focused on the effect of topically applied sunflower seed oil on skin condition, rates of nosocomial infections and mortality of preterm infants $\leq 34$ weeks gestational age. Treatment with sunflower seed oil $(n=51)$ resulted in a significant improvement in skin condition $(P=0.037)$ and a highly significant reduction in the incidence of nosocomial infections (Relative risk, 0.46 ; $95 \%$ confidence interval, $0.26-0.81 ; P=0.007)$ compared with infants not receiving topical prophylaxis $(n=52)$. Furthermore, there were no reported adverse events as a result of topical therapy. ${ }^{1}$

A subsequent randomized trial by Darmstadt et al. in Bangladesh, among hospitalized preterm infants born $\leq 33$ weeks, showed that topical sunflower seed oil resulted in a statistically significant $26 \%$ reduction in mortality rates, compared with infants not receiving topical emollient therapy (Hazard-adjusted ratio $0.74 ; 95 \%$ confidence interval $0.55-0.99 ; P=.042){ }^{4}$ Infants treated with sunflower seed oil were $41 \%$ less likely to develop nosocomial infections compared to controls (Adjusted incidence rate ratio 0.59, 95\% confidence interval $0.37-0.96 ; P=0.032) .^{5}$

Virgin coconut oil, like other plant oils, is composed of various fatty acids: medium chain triglycerides, consisting of 8 to 12 carbon atoms, and long chain fatty acids, containing 14 carbon atoms or more. The major medium chain triglyceride component of virgin coconut oil is lauric acid (12 carbon atoms), at $48 \%$ of its average fatty acid composition. Lauric acid has the most effective antimicrobial activity among these fatty acids. In addition, it has been demonstrated that it is the most stable for local and systemic utilization, and that its monoglyceride, monolaurin, is even more efficacious than lauric acid itself. Other medium chain triglycerides present in coconut oil, such as capric acid and monocaprin, have lower antimicrobial activity but may fortify the antimicrobial properties of lauric acid in coconut oil. ${ }^{6}$

Enig stated that monolaurin is the antiviral, antibacterial and antiprotozoal monoglyceride used by humans to destroy lipid-coated viruses such as human immunodeficiency virus (HIV), herpes, influenza, and cytomegalovirus; various pathogenic bacteria including Listeria monocytogenes and Helicobacter pylori; and the protozoan Giardia lamblia. ${ }^{7}$

In addition, a 6-month initial study on monolaurin and coconut oil carried out by Conrado Dayrit, a noted Filipino pharmacologist, on 15 HIV-positive patients showed a decrease in the viral counts in more than half of the patients and an improvement in their immune status as evidenced by an increase in their CD4 cell counts. This was the first clinical trial that built on Enig's statements on the anti-HIV attributes of coconut oil. ${ }^{6}$

In 2001, an in vitro analysis by Bergsson and associates, verified the gram-positive killing effect of fatty acids and monoglycerides. Lauric acid and monocaprin notably diminished the number of colony-forming units of Group A and B streptococci. Electron microscopy in addition elucidated that the bacteria are killed by disintegration of the cell membrane by the lipid. ${ }^{8}$

Although already being touted a miracle oil, the potential utility of virgin coconut oil as an antimicrobial agent remains to be fully appraised.

This study was undertaken to determine the efficacy of topically applied virgin coconut oil in the prevention of nosocomial infection in neonates born $\leq 34$ weeks gestational age. The specific objective was to determine if there is a difference in infection rates, need for DVET, all-cause mortality rates and length of hospital stay, among preterm neonates $\leq 34$ weeks gestational age who received $4 \mathrm{~g}$ per $\mathrm{kg}$ of body weight per treatment of virgin coconut oil applied topically twice a day for 14 days compared to those who did not receive topically applied virgin coconut oil.

The hypothesis was: topical application of virgin coconut oil in neonates born $\leq 34$ weeks gestational age will decrease the risk for nosocomial infections by $50 \%$.

\section{Methods}

\section{A. Study Design}

This study was a randomized, non-blinded, trial on topical virgin coconut oil using no treatment as the comparison group. 


\section{B. Study Site and Population}

Evaluation of the impact of topical therapy with virgin coconut oil on prevention of nosocomial infections in preterm infants was conducted at the NICU of the Philippine General Hospital, a tertiary, governmentsubsidized hospital that admits about 6,000 neonates annually with approximately $5 \%$ of which are born $\leq 34$ weeks gestational age.

All infants admitted to the NICU who were born $\leq 34$ weeks gestational age and $<72$ hours old were considered eligible for the study. Gestational age was determined by criteria of Ballard et al. ${ }^{9}$

The following infants were excluded: 1) infants with anticipated death within 48 hours of admission, for any reason, based on the admitting physician's assessment; 2) presence of a major congenital anomaly; 3) infants who required major surgical procedure; and 4) infant or mother with known immunodeficiency.

\section{Study Protocol}

\section{Randomization}

After obtaining informed parental consent, patients were stratified for gestational age and allocated to the treatment or control group using a table of random numbers. Randomization concealment was ensured by placing assignments in sealed opaque envelopes.

\section{Interventions and Procedures}

Infants in the intervention arm received TVCO twice daily for 14 days upon study entry, according to a dosing schedule that provided $4 \mathrm{~g}$ of oil per kilo of body weight per treatment. Patients in the control group received the standard of the unit for skin care, which is minimal to no use of topical emollients. Both groups received the same general care.

Baseline blood cultures were drawn routinely during the first 48 hours of admission on all patients, and these were compared with subsequent cultures of blood and cerebrospinal fluid (CSF) drawn for episodes of suspected sepsis. Cultures drawn for suspected sepsis that grew the same organism as present during baseline were considered persistent congenital infections and were excluded from analysis for nosocomial infection.

Infants enrolled in the study were followed up until 28 days of life or until discharge from the unit.

\section{Safety precautions}

The potential for contamination of the oil was minimized through the following measures: 1) daily supplies for each patient were distributed from a stock container stored at room temperature; 2) strict adherence to sterile procedures during dispensing and application; and 3) regular weekly replacement of the oil stock.

\section{Definition of terms}

Nosocomial infection is defined as a localized or systemic infection having an onset after birth, excluding those known to have been transmitted transplacentally and those that develop within 24-72 hours of birth..$^{10}$ This means that for most bacterial infections, signs and symptoms become evident 48 hours or more after admission. ${ }^{11}$

Clinical Sepsis is defined as the occurrence of the following signs and symptoms: temperature instability (hypothermia or hyperthermia), signs of organ hypoperfusion such as hypotension, delayed capillary refill time, decreased urine output or significant metabolic acidosis, apnea and/or bradycardia, leukocytosis or leukopenia, or thrombocytopenia, with a negative blood culture. ${ }^{11}$

Bacteriologically confirmed sepsis is defined as occurrence of any of the aforementioned signs and symptoms plus a recognized pathogen isolated from one or more blood cultures with no other recognized cause of infection. ${ }^{11}$

Pneumonia is defined as presence of at least one of the following: apnea, tachypnea, cyanosis, bradycardia, rhonchi, inability to feed plus radiologic evidence of new or progressive infiltrates, consolidation, or pleural effusion. ${ }^{11}$

Meningitis is defined as having at least one of the following signs and symptoms with no other recognized cause: fever, hypothermia, apnea, bradycardia, meningeal signs, cranial nerve signs, or irritability, in addition to at least one of the following: abnormal cerebrospinal fluid (CSF) findings, positive CSF gram stain or culture, positive blood culture or positive antigen test of CSF or blood. Meningitis is confirmed by examination of CSF obtained through lumbar puncture with the following criteria fulfilled: CSF cell count $>32$ leukocytes $/ \mathrm{mm}^{3}$ of which $>60 \%$ are neutrophils; CSF glucose $>50 \%$ of serum glucose; CSF protein of $>150 \mathrm{mg} / \mathrm{dl}$ with or without a positive CSF culture. ${ }^{11}$

Necrotizing enterocolitis (NEC) is defined as presence of microscopic or gross blood in stools with signs or symptoms of feeding intolerance and characteristic radiographic findings as defined by Bell's clinical staging for NEC. ${ }^{12}$

Urinary tract infection (UTI) is defined as pure culture of $>10^{5}$ organisms per $\mathrm{ml}$ from a specimen collected either by a midstream catch of urine or a suprapubic puncture. ${ }^{11}$

\section{Outcome measurements}

The primary outcome measure was the occurrence of nosocomial infections: sepsis whether clinical or bacteriologically proven, pneumonia, meningitis, NEC and UTI.

Secondary outcome measures included: 1) incidence of attending physician's performance of DVET for sepsis; 2) mortality rate; and 3) number of days to discharge. 


\section{E. Data Analysis}

All outcomes were analyzed by intention to treat using SPSS computer software. Means and standard deviations for continuous variables were compared via T-test. Chisquare was utilized for categorical variables. A $P$-value $<0.05$ was considered statistically significant.

For the primary and secondary outcomes, the relative risk (RR), relative risk reduction (RRR), absolute risk reduction (ARR) and number needed to treat (NNT) along with $95 \%$ confidence intervals were computed for categorical variables. For continuous variables, difference of means with $95 \%$ confidence interval was computed.

An interim analysis is scheduled after at least $20 \%$ of the subjects in each treatment group have been recruited. An incidence of any nosocomial infection or death at least 10\% higher in the experimental group compared to the control group was considered clinically significant enough to consider pretermination of the trial.

\section{F. Sample Size Calculation}

The study was designed to test the hypothesis that topical application of $\mathrm{VCO}$ will decrease the risk for nosocomial infection by $50 \%$ (i.e. a reduction from the current risk of $30 \%$ to $15 \%$. Using an alpha error of $5 \%$ and a beta error of $20 \%$, the sample size required to be able to prove the above hypothesis is 123 infants per intervention group.

\section{Ethical Considerations}

The study protocol was reviewed and approved by the Research Implementation and Development Office of the Philippine General Hospital. The study included neonates born $\leq 34$ weeks gestational age. The population is considered vulnerable but is justified as the intervention is expected to benefit preterm infants considering that they are at highest risk of developing sepsis. A research assistant provided information about the study to the parents of the patients. She was tasked to obtain written informed consent from the parents prior to the randomization of infants to the two study groups. She did not provide services to any subject. A definition of informed consent was included in the consent form. The informed consent form was read and explained to the parents in English and Filipino. The parents were given time to think and consult others before consent was given. The purpose of the study, the description of the study procedure, and the possible risks and benefits were likewise included in the consent form. Parents were informed of any adverse effects that may arise from the infant's participation in the study and how it will be managed. They were informed of their right to refuse and withdraw from the trial anytime without any change in the care given to the baby. Anonymization of patient data, removal of personal identifiers and security of databases was ensured. Parents were assured of the privacy and confidentiality of information obtained from their baby that are required by the trial.

\section{Results}

A total of 74 patients were considered as potential study participants (through their parents/guardians), 65 satisfied the inclusion and exclusion criteria and 52 were included after informed consent was obtained from their parents and guardians.

After the completion of the participation of the $52^{\text {nd }}$ patient (such that $42 \%$ of the target sample size was already achieved), an interim analysis was done as scheduled.

Of the 52 patients, 28 infants were randomized to receive routine skin care and 24 infants randomized to receive topically applied virgin coconut oil. Descriptive variables for these study subjects are shown in Table 1.

Table 1. Baseline characteristics of patients.

\begin{tabular}{lcc}
\hline & $\begin{array}{c}\text { Routine Skin } \\
\text { Care Group } \\
\mathbf{( N = 2 8 )}\end{array}$ & $\begin{array}{c}\text { Topical Virgin } \\
\text { Coconut Oil } \\
\text { Group (N=24) }\end{array}$ \\
\hline Male sex & $16(57)$ & $15(62)$ \\
Mean birth weight $(\mathrm{g})$ & $1212.50 \pm 390.07$ & $1326.00 \pm 318.56$ \\
Mean birth length $(\mathrm{cm})$ & $38.21 \pm 4.53$ & $40.04 \pm 3.74$ \\
Mean birth head circumference $(\mathrm{cm})$ & $26.76 \pm 2.54$ & $28.10 \pm 2.58$ \\
Apgar score at 1 minute $<7$ & $9(32)$ & $6(25)$ \\
Apgar score at 5 minutes $<7$ & $4(14)$ & $2(8)$ \\
Maternal infection & $7(25)$ & $9(38)$ \\
Oxygen support or mechanical & $18(64)$ & $13(54)$ \\
ventilation & & \\
Umbilical vessel cannulation & $12(43)$ & $5(21)$ \\
\hline Data are expressed as number $(\%)$ unless otherwise stated &
\end{tabular}

The groups were similar in terms of proportion of male infants and anthropometrics at birth (weight, length and head circumference). Apgar scores between the two groups were also comparable. Furthermore, there were no statistically significant differences between the two groups with respect to maternal history of infection, need for oxygen support or mechanical ventilation, and performance of umbilical vessel cannulation.

The primary outcome measure for this clinical trial was the incidence of nosocomial infections: sepsis whether clinical or bacteriologically proven, pneumonia, meningitis, NEC and UTI. There were no episodes of meningitis, NEC and UTI in both treatment groups. The occurrence of nosocomial sepsis and pneumonia among the study groups is shown in Table 2.

The frequency of nosocomial sepsis in the routine skin care group was higher compared to the topical virgin coconut oil group (12/28 [43\%] versus 9/24 [38\%]). This difference however, was not significant $(P=0.695)$. The incidence of nosocomial pneumonia was higher in the topical oil group (4/24 [17\%]) compared to the control group $(2 / 28[7 \%])$, but this was also not statistically significant $(P=0.284)$. 
Table 2. Results

\begin{tabular}{|c|c|c|c|}
\hline & $\begin{array}{c}\text { Routine } \\
\text { Skin Care } \\
\text { Group } \\
(\mathbf{N}=\mathbf{2 8})\end{array}$ & $\begin{array}{c}\text { Topical } \\
\text { Virgin } \\
\text { Coconut } \\
\text { Oil Group } \\
(\mathrm{N}=\mathbf{2 4 )}\end{array}$ & $\begin{array}{c}P- \\
\text { value }\end{array}$ \\
\hline Nosocomial Sepsis & $12(43)$ & $9(38)$ & 0.695 \\
\hline Nosocomial Pneumonia & $2(7)$ & $4(17)$ & 0.284 \\
\hline $\begin{array}{l}\text { Decision to do double-volume } \\
\text { exchange transfusion for sepsis }\end{array}$ & $3(11)$ & 0 & 0.098 \\
\hline Mortality & $4(14)$ & $3(12)$ & 0.851 \\
\hline Mean length of hospital stay (days) & $14.00 \pm 4.14$ & $13.91 \pm 2.81$ & 0.629 \\
\hline
\end{tabular}

The decision to do DVET for sepsis was made by the attending physicians of 3 infants in the control group, whereas no such order was done among those in the treatment group. This difference however, was not statistically significant $(P=0.098)$.

Mortality rates between groups were comparable; with the routine skin care group having a $14 \%$ mortality rate and the topical virgin coconut oil group having a $12 \%$ mortality rate $(P=0.851)$.

Lastly, there was also no significant difference in the mean length of hospitalization, with control subjects staying $14.00 \pm 4.14$ days versus topical virgin coconut oil subjects staying $13.91 \pm 2.81$ days $(P=0.629)$

The relative risk (RR), relative risk reduction (RRR), absolute risk reduction (ARR) and number needed to treat (NNT) along with 95\% confidence intervals for categorical variables among the trial outcomes are summarized in Table 3. For length of hospital stay, the difference of means with $95 \%$ confidence interval is shown.

Table 3. Point estimates with $95 \%$ confidence intervals of effect of topical Virgin Coconut Oil among preterm neonates

\begin{tabular}{lcccc}
\hline & RR & RRR & ARR & NNT \\
\hline Nosocomial Sepsis & 0.875 & 0.125 & 0.054 & 19 \\
& {$[0.447$,} & {$[-0.711$,} & {$[-0.213$,} & \\
& $1.711]$ & $0.553]$ & $0.320]$ & \\
Nosocomial Pneumonia & 2.333 & -1.333 & -0.095 & -11 \\
& {$[0.468$,} & {$[-0.532$,} & {$[-0.082$,} & \\
& $11.644]$ & $10.644]$ & $0.272]$ & \multirow{2}{*}{0.101} \\
Decision to do double- & 0.166 & 0.834 & & \\
volume exchange & & & & \\
transfusion for sepsis & {$[0.009$,} & {$[-2.056$,} & {$[-0.030$,} & \\
& $3.056]$ & $0.991]$ & $0.231]$ & \multirow{2}{*}{0.018} \\
Mortality & 0.875 & 0.125 & {$[-0.167$,} & \\
& {$[0.217$,} & {$[-2.528$,} & $0.203]$ & \\
& $3.528]$ & $0.783]$ & Difference of Means & \\
Length of hospital stay & & $0.091[-2.338,2.520]$ & \\
\hline
\end{tabular}

\section{Discussion}

The interim analysis of this clinical trial showed a decrease of $12.5 \%$ of the baseline risk for nosocomial sepsis in the topical virgin coconut oil group. Compared to the control group, the risk for nosocomial sepsis in the treatment group was reduced by $5.4 \%$. For every 19 preterm infants who received topically applied virgin coconut oil, one case of nosocomial sepsis may have been prevented. This difference however, was not statistically significant.

A drop of $12.5 \%$ of the baseline risk for mortality was likewise demonstrated for the topical virgin coconut oil group, with a small decline in the risk of dying (1.8\%) compared to the routine skin care group. For every 56 premature infants given virgin coconut oil topical application, one may have been prevented from dying. However, this observation was also not statistically significant.

A trend toward decreased need for DVET for sepsis was found in the treatment group $[R R=0.166[0.009,3.056]$. There were 3 cases in the routine skin care group with sepsis that were considered eligible for DVET whereas none of the sepsis cases in the virgin coconut oil group were severe enough to warrant such therapy. The decision to do DVET for sepsis was made by the attending physicians based on a positive blood culture (2 cases of Enterobacter cloacae and 1 case of Klebsiella pneumoniae) and progression of clinical signs and symptoms of sepsis. While not statistically significant, this suggests that the clinical severity of disease may be attenuated by topical virgin coconut oil therapy.

There were 2 cases of nosocomial pneumonia in the control group versus 4 cases in the treatment group. This translates to an increase of $9.5 \%$ in the risk of occurrence of nosocomial pneumonia in the experimental group. With a $95 \%$ confidence interval of -0.082 to 0.272 , data is insufficient to state whether harm truly exists. However, this difference was already considered clinically significant enough to consider pretermination of the trial due to the possibility of harm.

The mean length of hospital stay was minimally shorter by 0.091 days in those infants who received topical virgin coconut oil compared to the control group $(P=0.629)$.

Previous studies have highlighted the possible antimicrobial effects of virgin coconut oil, particularly its constituent fatty acids lauric acid and capric acid, and their corresponding monoglycerides monolaurin and monocaprin. 6,7 There is a paucity of data however, regarding effectiveness against gram-negative bacteria, which remain to be the top causes of nosocomial infections in our setting. This may explain the small difference in the rate of nosocomial infections seen in this trial. In this light, in vitro studies may be undertaken to elucidate the usefulness of virgin coconut oil in the management of specific gramnegative bacterial infections.

Thormar and his partners illustrated that capric and lauric acids are active in killing Candida albicans in vitro and demonstrated that even if these lipids are toxic in cell cultures, they are not harmful to skin and mucosa at higher concentrations. ${ }^{13}$ However, in the course of this trial, none of the subjects in either treatment group developed fungal 
sepsis. It remains to be seen whether the in vitro properties of coconut oil against Candidal infections translate to clinical significance in preterm neonates.

Power calculations were done as part of this interim analysis (Alpha $=0.212, \mathrm{P}_{0}=0.43, \mathrm{P}_{1}=0.38$ ). The sample size of 28 in the control group and 24 in the treatment group resulted in a Power $=0.0299$. The power of the test to determine a difference in the outcome of the two groups using the above sample size is only $3 \%$. Meaning, there is a $97 \%$ chance of incorrectly accepting the hypothesis that there is no significant difference in the incidence of nosocomial sepsis in the two groups. Increasing the power of the study may well uncover a trend of benefit for the use of topically applied virgin coconut oil in the prevention of sepsis in premature infants. However, based on the incidence of nosocomial sepsis observed $(43 \%$ in the control group and $38 \%$ in the treatment group), the sample size required was recomputed at over 2,000 subjects per treatment arm in order to detect a statistically significant difference in the incidence of nosocomial sepsis in the groups. A much larger trial than originally planned may be necessary to demonstrate a reduction in nosocomial sepsis with the use of topical VCO in premature infants.

Preliminary results of an unpublished randomized controlled trial done by Amante et al. at the Philippine General Hospital in 2004, designed to detect the effect of virgin coconut oil supplementation to enteral feeds on the weight gain of neonates weighing $\leq 1,500 \mathrm{~g}$ at birth, demonstrated a decrease in the incidence of sepsis in the treatment group at $7.1 \%$ versus $36.4 \%$ in the control group. ${ }^{14}$ Although also not statistically significant, this was a much larger effect on the rate of nosocomial sepsis than the 5.4\% decrease seen in this trial on topically applied virgin coconut oil. It appears that the route of administration may be an important factor in the effectiveness of virgin coconut oil in the prevention of infections.

Based on these considerations, this clinical trial was stopped before the computed sample size was reached. The authors deemed it impractical and unethical to recruit an additional 2,000 infants per treatment group given that the clinical benefits of topical application of virgin coconut oil in premature infants may not considerably outweigh possible adverse outcomes (i.e. nosocomial pneumonia). Oral administration of virgin coconut oil is easier than topical application, with the added benefit of augmenting caloric intake. The authors recommend that future studies on the use of virgin coconut oil in neonatal medicine would be better directed towards oral rather than topical administration.

\section{Conclusion and Recommendation}

This study compared the effect of topical virgin coconut oil versus routine skin care in infants born $\leq 34$ weeks of gestation. There was insufficient evidence to conclude there was a difference in the incidence of nosocomial infections, mortality rates and length of hospital stay between the two treatment groups. There was a trend for decreased need for DVET for sepsis in infants who received topical virgin coconut oil. There was however a possibility of harm, with an increased incidence of nosocomial pneumonia in the topical coconut oil group. It is recommended that subsequent clinical investigations on the use of virgin coconut oil in newborns focus on the utility and safety of orally rather than topically administered virgin coconut oil in the prevention of infections in premature infants.

\section{References}

1. Darmstadt GL, Badrawi N, Law PA, et al. Topically applied sunflower seed oil prevents invasive bacterial infections in preterm infants in Egypt: a randomized, controlled clinical trial. Pediatr Infect Dis J. 2004; 23(8):719-25.

2. Darmstadt GL, Mao-Qiang M, Chi E, et al. Impact of topical oils on the skin barrier: possible implications for neonatal health in developing countries. Acta Paediatr. 2002; 91(5):546-54

3. Conner JM, Soll RF, Edwards WH. Topical ointment for preventing infection in preterm infants. Cochrane Database Syst Rev. 2004; (1):CD001150

4. Darmstadt GL, Saha SK, Ahmed AS, et al. Effect of skin barrier therapy on neonatal mortality rates in preterm infants in Bangladesh: a randomized, controlled, clinical trial. Pediatrics. 2008; 121(3):522-9.

5. Darmstadt GL, Saha SK, Ahmed AS, et al. Effect of topical treatment with skin barrier-enhancing emollients on nosocomial infections in preterm infants in Bangladesh: a randomised controlled trial. Lancet. 2005; 365(9464):1039-45.

6. Dayrit CS. Coconut oil in health and disease: its and monolaurin's potential as cure for HIV/AIDS [Online]. 2000 [cited 2012 Nov]. Available from http://www.coconutresearchcenter.org/article10526.pdf

7. Enig MG. Coconut: In support of good health for the $21^{\text {st }}$ century [Online]. 1999 [cited 2012 Nov]. Available from http://www.coconutresearchcenter.org/article10147.htm

8. Bergsson G, Arnfinnsson J, Steingrímsson O, Thormar H. Killing of gram-positive cocci by fatty acids and monoglycerides. APMIS. 2001; 109(10):670-8.

9. Ballard JL, Khoury JC, Wedig K, Wang L, Eilers-Walsman BL, Lipp R. New Ballard Score, expanded to include extremely premature infants. J Pediatr. 1991; 119(3):417-23.

10. Hernandez EA, ed. Standards of Newborn Care, $2^{\text {nd }}$ ed. Section of Neonatology of the Philippine Pediatric Society, Inc.; 2001.

11. Bravo LC, et al. A Guide to Pediatric Infection Control. Manila: Philippine General Hospital; 2001.

12. Teaching Files: Necrotizing Enterocolitis [Online]. 2000 [cited 2012 Nov]. Available from http://www.neonatology.org.

13. Bergsson G, Arnfinnsson J, Steingrímsson O, Thormar H. In vitro killing of Candida albicans by fatty acids and monoglycerides. Antimicrob Agents Chemother. 2001; 45(11):3209-12.

14. Amante MR, Mantaring JV. Randomized controlled trial on the use of virgin coconut oil supplement in augmenting weight gain among infants less than 35 weeks gestation. 2004. Unpublished. 